\title{
Coexistence of Carcinoma and Tuberculosis in the Breast: A Rarity
}

\author{
Ketan Vagholkar ${ }^{*}$, Abhijit Budhkar1, Indumati Gopinathan² \\ ${ }^{1}$ Dr. D. Y. Patil Medical College, Navi, Mumbai, India \\ ${ }^{2}$ Clinicopathological Laboratory, Mumbai, India \\ Email: "kvagholkar@yahoo.com
}

Received 22 November 2014; revised 21 December 2014; accepted 2 January 2015

Copyright $@ 2015$ by authors and Scientific Research Publishing Inc.

This work is licensed under the Creative Commons Attribution International License (CC BY).

http://creativecommons.org/licenses/by/4.0/

c) (i) Open Access

\begin{abstract}
Background: Malignancy of the breast is one of the commonest lesions of the breast. Amongst the infective lesions tuberculosis has now become a common occurrence especially in the developing world. However, co-existence of the two in the same breast is extremely rare. Introduction: Since both of these lesions have a different natural history, co-existence of tuberculosis and malignancy poses a diagnostic dilemma to both the surgeon and the pathologist. Awareness of the co-existence of the two lesions in the same patient is pivotal for early diagnosis and planning the management. Case Report: In view of its rarity, a case of coexistence of the two diseases in the same beast is reported in order to create an awareness of their co-existence. Discussion: The diagnostic implications and management are discussed. Conclusion: Awareness of the co-existence of these divergent pathological conditions is essential for early diagnosis and planning of an accurate treatment protocol.
\end{abstract}

\section{Keywords}

Tuberculosis, Carcinoma, Breast, Coexistence

\section{Introduction}

Tuberculosis is one of the commonest chronic infections affecting primarily the lungs, lymph nodes and intestines. Tuberculosis of the breast once an uncommon disease is now becoming common especially in the developing countries [1]. Concomitant upsurgence in the incidence of malignancy of the breast is by itself a problem confronting surgeons. Coexistence of both the lesions in the same breast is extremely uncommon. However if present together, it may pose a diagnostic dilemma to the surgeon. Awareness of coexistence of these two lesions is pivotal in avoiding delay in commencing treatment thereby reducing the morbidity and mortality associ- 
ated with these conditions. A case of coexistence of both tuberculosis and malignancy in same breast is presented here along with a review of literature.

\section{Case Report}

58-year-old lady was referred to our surgical unit for a mass in her right breast. She gave a history of having undergone two surgical procedures on the same breast for a cystic lesion which was diagnosed as fibrocystic disease histologically on two different occasions. However the lump redeveloped in the same breast and increased in size over a period of time. Patient did not have any systemic symptoms. There was no history of tuberculosis or any contact with a patient suffering from tuberculosis in either the family or in close vicinity.

Physical examination revealed a large mass approximately $6 \mathrm{~cm}$ in diameter involving almost the entire right breast. The overlying nipple areola complex was scarred with evidence of ulceration (Figure 1). The mobility of mass within the breast and on the underlying muscle was restricted. Ipsilateral axilla did not reveal any lymphadenopathy clinically. Contralateral breast and axilla were normal. There was no evidence of cervical lymphadenopathy or spinal tenderness. Respiratory system did not reveal any abnormality. FNAC was done and confirmed the diagnosis of invasive ductal carcinoma. Special staining of the blood stained fluid from the ulcerated area revealed the presence of acid fast bacteria (Figure 2). Metastatic work up did not reveal any metastasis. Patient underwent two cycles of chemotherapy (CMF regime) preoperatively in order to downstage the locally advanced lesion. 25\% regression in the size of the mass was observed after chemotherapy. Patient was then subjected to modified radical mastectomy with axillary clearance.

Histological evaluation of the specimen confirmed the diagnosis of invasive ductal carcinoma (Figure 3). However, in addition, granulomas comprising of outer rim of epitheloid cells typical of tuberculosis were seen in a few sections (Figure 4). Histopathological examination of axillary lymphnodes revealed positive evidence of metastasis in the lymph nodes. However there was no evidence of any granuloma. Post-operative recovery

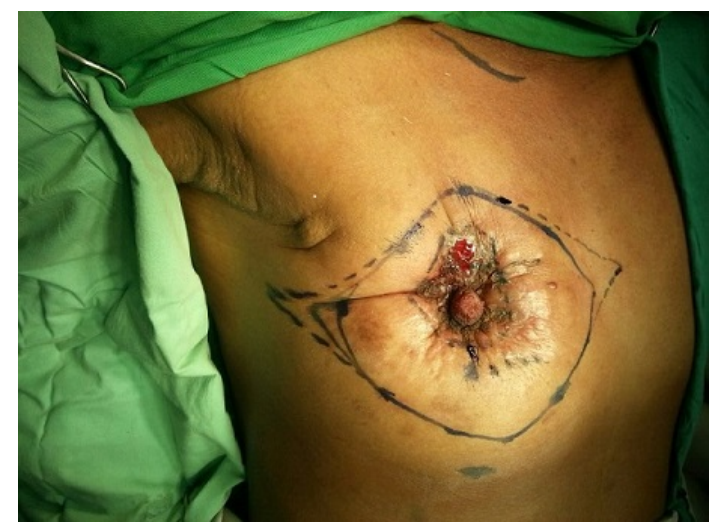

Figure 1. Pre-operative photograph of the affected breast showing extensive scarring and ulceration.

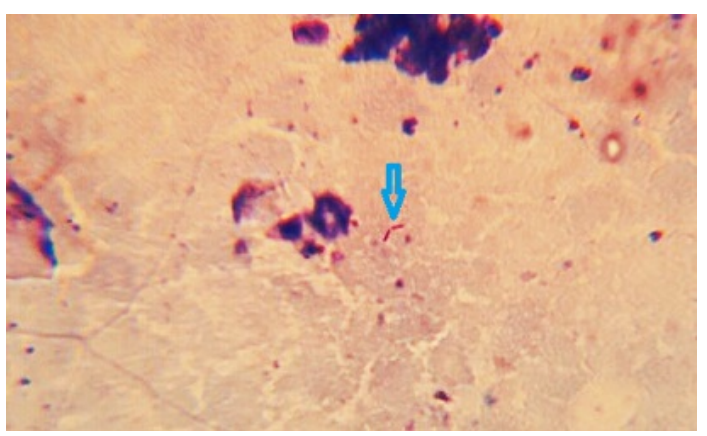

Figure 2. Acid fast bacteria marked by the blue arrow seen in the discharge from the ulcerated area (Zeihl Nelson staining, magnification $\times 100$ ). 


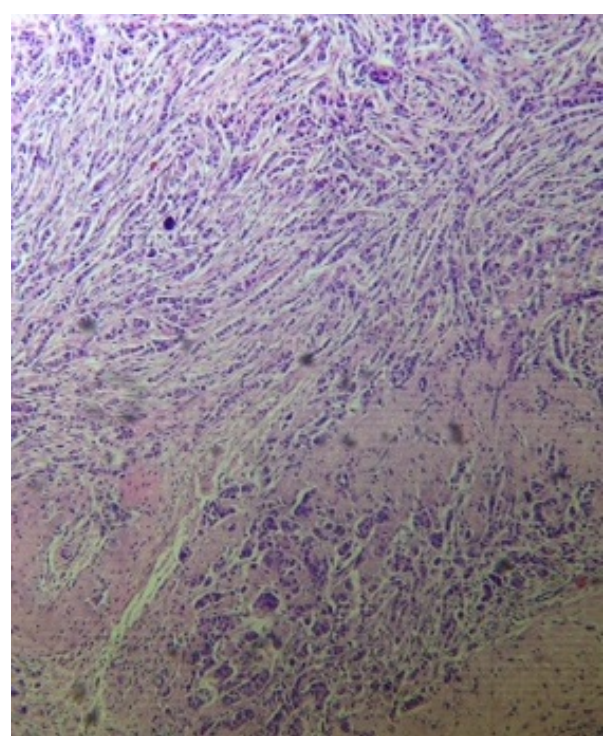

Figure 3. Infiltrating tumor cells (Hematoxylin \& Eosin staining, magnification $\times 10$ ).

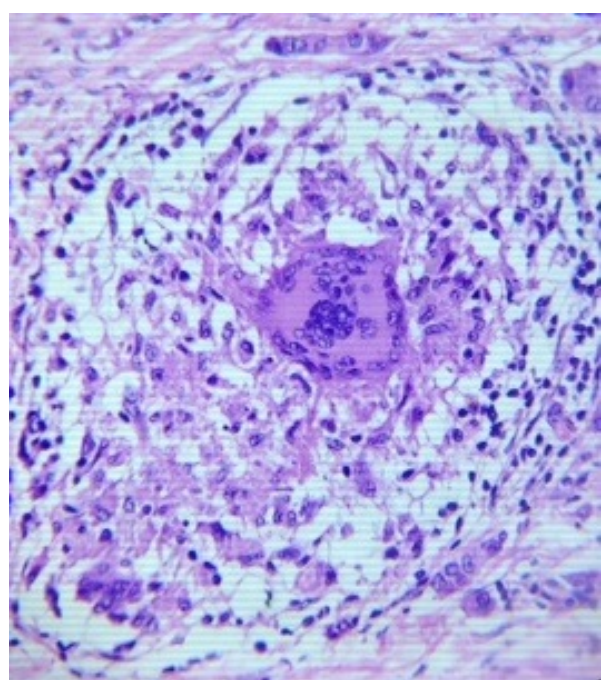

Figure 4. Lymphoepitheloid granulomas amidst malignant cells (Hematoxylin \& Eosin staining, magnification $\times 40$ ).

was uneventful. Patient has completed 4 cycles of chemotherapy and is presently undergoing local irradiation. A 6-month course of medications for tuberculosis has been completed with no side effects. This was commenced after the completion of chemotherapy cycles as the patient experienced severe systemic complications with anticancer chemotherapy drugs.

\section{Discussion}

Tuberculosis of the breast is traditionally described as a great masquerader [1]-[4]. This is because it may present with a mass to begin with or a septic lesion in the form of an abscess. Either of them eventually ends up with the formation of a sinus. It can cause deformity of nipple areola complex as well as extensive scarring of the overlying breast. A scarred breast or a grossly deformed nipple areola complex as was seen in the case presented should raise the suspicion of tuberculosis. However these findings closely simulate malignancy making diagnosis extremely difficulty. Lump in the breast always merits prompt investigation by an FNAC. FNAC has 
high sensitivity and specificity in the diagnosis of breast cancer. However the diagnostic efficacy of FNAC in tuberculosis of breast is limited. Presence of epitheloid cells may be suggestive but in majority of cases these cells are usually not picked up [3]. Presence of caseating granulomas is diagnostic of TB but this necessitates elaborate histopathological evaluation [4]. Detection of acid fast bacilli in the lesions on the tissue culture or in discharge is diagnostic of tuberculosis [1]. In the case presented AFB were detected on examination of the blood stained discharge from the ulcerated area (Figure 2). However in practice many a times it is difficult to detect acid fast bacilli in the lesion. Review of articles on Google scholar and PubMed databases have revealed very few number of cases describing coexistence of tuberculosis and cancer in same breast. The coexistence of tuberculosis of the axillary lymph nodes along with breast cancer supersedes the incidence of coexistence of tuberculosis and malignancy in same breast numerically [3]-[6]. The exact cause effect relationship continues to pose a dilemma to the pathologist. Whether it is chronic inflammation due to tuberculosis which induces malignant transformation is yet to be researched. Various permutations and combinations of coexistence of tuberculosis and breast cancer have been reviewed. In one such review by Akbulut et al., 29 cases were reported till year 2011 as per PubMed and Google scholar databases [7]. 21 of these patients had breast cancer along with tuberculosis of the axillary lymph nodes. These patients did not have tuberculosis in the breast. Out of remaining 8 patients, 5 had both tuberculosis of breast and cancer. Both tumor metastasis and tuberculosis were present in dissected axillae of 14 patients. Amongst these 14 patients, 6 had both cancer metastasis and tuberculosis detected in same lymphnodes. X-ray of the local parts especially the axilla has been described as a diagnostic tool in suspected lesions [8]. Calcification of the lymphnodes is highly suggestive of tuberculosis. However in the case presented, calcification was not present on the chest X-ray in region of axilla and breast [9]. In cases of co-existence of two lesions it is advisable to commence treatment for the malignant lesion [10]. This may involve surgical intervention along with neoadjuvant therapy. A diagnosis of malignancy is pivotal as radical surgery can be performed without delay especially in early stages. Tuberculosis by itself does not require surgical intervention. Few patients develop serious side effects from chemotherapeutic drugs necessitating temporary cessation of therapy. It is therefore a safe practice to commence antituberculosis treatment after completion of cancer chemotherapy treatment. This can avoid morbid complications associated with either group of drugs. Tuberculosis needs to be treated by a combination of drugs. A four drug regime for 2 months followed by a two drug regime for 4 months is sufficient for cure. Prognosis of breast cancer patients with concomitant tuberculosis needs further evaluation. There are no studies which have addressed this issue.

Patients suffering from tuberculosis usually have depressed cell mediated immunity (CMI). Whether depressed CMI has an adverse impact on 5-year survival in such patients needs further evaluation. Data on this issue is lacking as number of lesion with coexistence of tuberculosis and malignancy of breast is extremely small.

\section{Conclusions}

Coexistence of carcinoma and tuberculosis of breast or axillary lymph nodes is extremely rare.

Gross deformity of breast with or without septic lesions should raise a suspicion of tuberculosis.

Elaborate histopathological evaluation of the specimen by a pathologist who is aware of this coexistence can only help in establishment of the diagnosis of this lethal duo.

\section{Acknowledgements}

We would like to thank Dr. Shirish Patil, Dean of Dr. D. Y. Patil Medical College, Navi Mumbai, India for allowing us to publish this case report. We would also like to thank Mr. Parth K. Vagholkar for his help in typesetting the manuscripts. Written informed consent was obtained from the patient for publication of this case report and accompanying images.

\section{Conflict of Interests}

The authors declare that they have no competing interests.

\section{References}

[1] Vagholkar, K., Gopinathan, I., Pandey, S. and Maurya, I. (2014) Tuberculosis of the Breast (Case Report and Review of Literature). Internet Journal of Surgery, 31. 
[2] Gon, S., Bhattacharya, A., Maunder, B. and Kundu, S. (2013) Tubercular Mastitis-A Great Masquerade. Turkish Journal of Pathology, 29, 61-63.

[3] Shinde, S.R., Chandawarkar, R.Y. and Deshmukh, S.P. (1995) Tuberculosis of the Breast Masquerading as Carcinoma: A Study of 100 Patients. World Journal of Surgery, 19, 379-381. http://dx.doi.org/10.1007/BF00299163

[4] Prathima, S., Kalyani, R. and Parimala, S. (2014) Primary Tubercular Mastitis Masquerading as Malignancy. Journal of Natural Science, Biology and Medicine, 5, 184-186. http://dx.doi.org/10.4103/0976-9668.127324

[5] Pandey, M., Abraham, E.K., Chandramohan, K. and Rajan, B. (2003) Tuberculosis and Metastic Co-Existence in Axillary Lymph Node: A Case Report. World Journal of Surgical Oncology, 1, 3. http://dx.doi.org/10.1186/1477-7819-1-3

[6] Alzaraa, A. and Dalal, N. (2008) Co-Existence of Carcinoma and Tuberculosis in One Breast. World Journal of Surgical Oncology, 6, 29. http://dx.doi.org/10.1186/1477-7819-6-29

[7] Akbulut, S., Sogutcu, N. and Yagmur, Y. (2011) Coexistence of Breast Cancer and Tuberculosis in Axillary Lymph Nodes: A Case Report and Literature Review. Breast Cancer Research and Treatment, 130, 1037-1042. http://dx.doi.org/10.1007/s10549-011-1634-8

[8] Baslaim, M.M., Al Amoudi, S.A., Al Ghamdi, M.A., Ashour, A.S. and Al Numani, T.S. (2013) Case Report: Breast Cancer Associated with Contralateral Tuberculosis of Axillary Lymph Nodes. World Journal of Surgical Oncology, 11, 43. http://dx.doi.org/10.1186/1477-7819-11-43

[9] Fujii, T., Kimura, M., Yanagita, Y., Koida, T. and Kuwano, H. (2003) Tuberculosis of Axillary Lymph Nodes with Primary Breast Cancer. Breast Cancer, 10, 175-178. http://dx.doi.org/10.1007/BF02967646

[10] Gaurav, M., Rajagopal, S., Mary, M. and Bhavatej, E. (2008) Concomitant Tuberculosis and Metastasis in Axillary Lymph Nodes: A Diagnostic Surprise. ANZ Journal of Surgery, 7899, 825-856. 
Scientific Research Publishing (SCIRP) is one of the largest Open Access journal publishers. It is currently publishing more than 200 open access, online, peer-reviewed journals covering a wide range of academic disciplines. SCIRP serves the worldwide academic communities and contributes to the progress and application of science with its publication.

Other selected journals from SCIRP are listed as below. Submit your manuscript to us via either submit@scirp.org or Online Submission Portal.
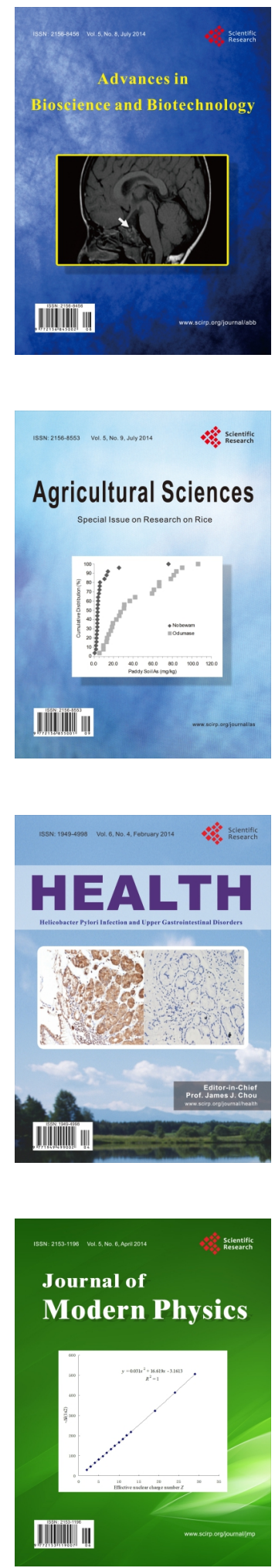
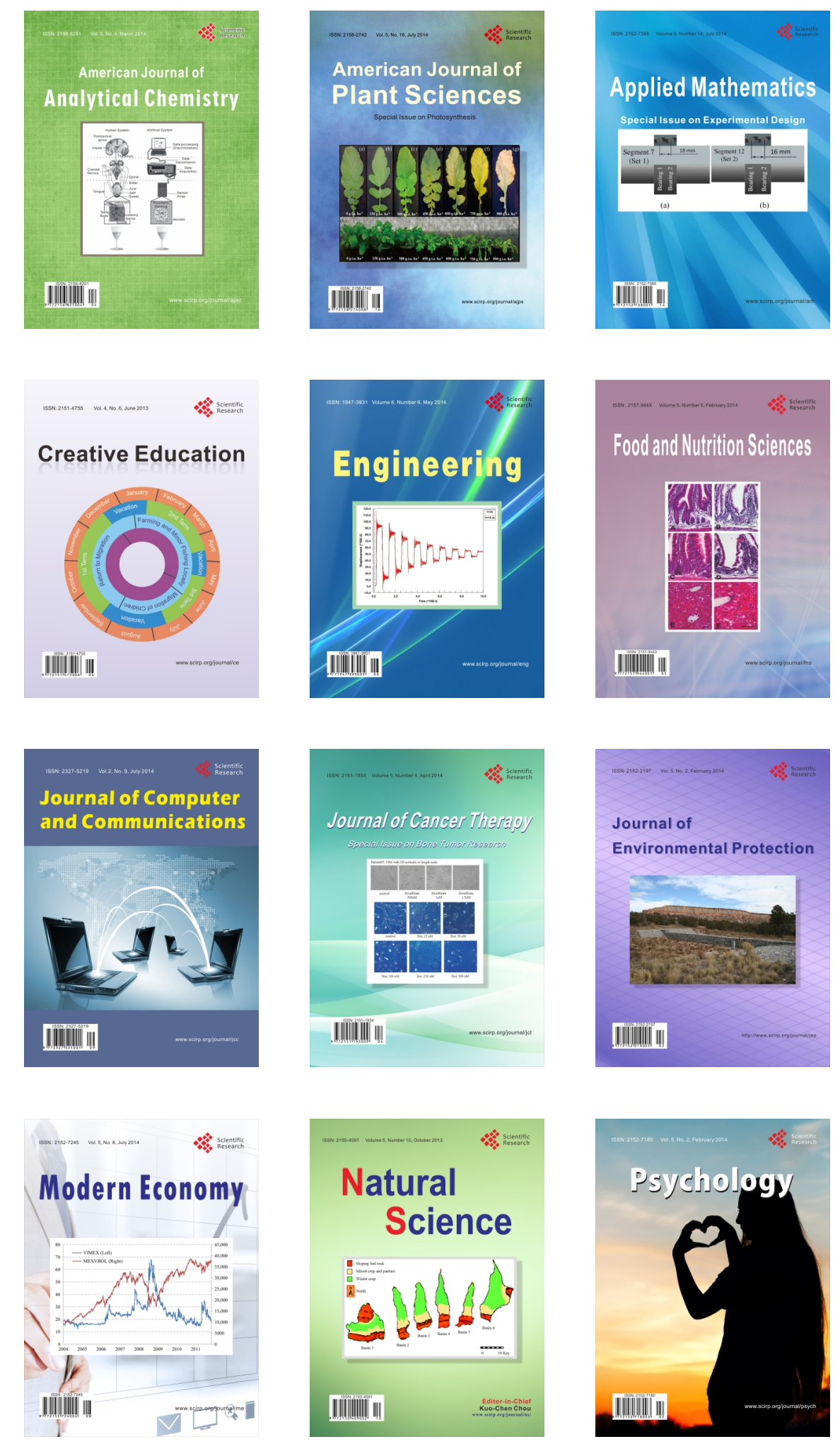